\title{
A non-uniformly moving line force in an anisotropic elastic solid
}

\author{
By K UAN G-CHONG W U \\ Institute of Applied Mechanics, National Taiwan University, Taipei 106, Taiwan
}

Received 18 May 2001; accepted 31 October 2001; published online 14 May 2002

The transient motion due to a line load moving through an unbounded anisotropic elastic body is considered. The force is applied suddenly in the body, and then moves off with non-uniform speed. Explicit expressions for the displacements are derived using the reciprocal theorem. A parametric form of the Mach wavefronts is presented when the force moves in a fixed direction, and first-motion responses are obtained for different wave arrivals by asymptotic methods.

Keywords: moving force; anisotropic solids; elastic wave propagation

\section{Introduction}

The problem of a moving force in an elastic body has been of interest in such areas as structural dynamics and seismology. Early work on moving forces includes solutions for the steady motion of a point force in an unbounded elastic body by Eason et al. (1956) and for a line force on an elastic half-space by Sneddon (1951) and Cole \& Huth (1958). The transient problem of a force that is applied suddenly on the surface at some instant and travels with constant velocity was studied by Ang (1960) and Payton (1967) for a line force and by Gakenheimer \& Miklowitz (1969) for a point force. The epicentre motion of an elastic half-space due to a buried moving line force was treated by Payton (1968). The wave motion resulting from a line force moving non-uniformly in a fixed direction was considered in Freund (1972) for an unbounded solid and in Freund (1973) for a half-space. The aforementioned works are for isotropic materials.

The steady motion due to a line force in an unbounded anisotropic elastic solid was studied by Stroh (1962). It appears that the transient motion induced by a moving force in an anisotropic solid has not been considered so far. The objective of the present paper is thus to provide an exact solution to the transient problem for materials of general anisotropy. The force is assumed to be applied suddenly at the origin of a Cartesian coordinate system $\left(x_{1}, x_{2}\right)$ and then to move in an arbitrary manner.

Most of the works on transient motion employed integral transform methods and the Cagniard-de Hoop inversion technique. An alternative and simpler approach was used by Payton (1967), in which the solution to the moving-force problem was obtained by applying the reciprocal theorem in conjunction with the solution to a stationary-force problem. Although Payton considered only the uniform force motion, 
his approach can be extended and is in fact used here to study arbitrary non-uniform motion. The solution to the stationary problem used is that given by $\mathrm{Wu}(2000)$. The solution was derived with a formulation which is based on the solution to an eigenvalue problem. The displacement fields are derived in the form of an integral over the history of the motion. For the special case of a uniformly moving force in a fixed direction, the particle velocities are obtained in closed form.

In discussing the transient motion it is essential to determine the wavefronts generated by the moving force. The wavefronts consist of bulk waves and possible Mach waves when the force moves supersonically. Freund (1972) constructed the Mach wavefronts by integrating the bicharacteristic equations for isotropic solids. In the present paper an explicit parametric form of the Mach waves for general anisotropic solids is obtained by considering the wavefront as the envelopes of the wavelets which are emitted as the force moves along the $x_{1}$-axis. Examples of accelerating and decelerating forces as considered by Freund (1972) for an isotropic body are presented for a cubic material. First-motion responses are derived for different wave arrivals by asymptotic methods.

The paper is laid out as follows: in $\S 2$ basic equations in a formulation proposed by $\mathrm{Wu}$ are outlined. The solution of an impulsive line force is also given in $\S 2$. In $\S 3$ the displacements due to a non-uniformly moving force are derived. The wavefronts are discussed in $\S 4$. An asymptotic wavefront analysis is presented in $\S 5$. The special case of a uniformly moving force is considered in $\S 6$. Finally, some concluding remarks are given.

\section{Basic equations}

For two-dimensional deformation in which the Cartesian components of the stress $\sigma_{i j}$ and the displacement $u_{i}, i, j=1,2,3$, are independent of $x_{3}$, the general dynamic self-similar solution may be represented as (Wu 2000)

$$
\begin{aligned}
\dot{\boldsymbol{u}}\left(x_{1}, x_{2}, t\right) & =-2 \operatorname{Re}\left\{\boldsymbol{A}(\boldsymbol{\omega})\left\langle\omega_{*} \frac{\partial \omega_{*}}{\partial x_{1}}\right\rangle \boldsymbol{f}(\boldsymbol{\omega})\right\}, \\
\boldsymbol{t}_{2}\left(x_{1}, x_{2}, t\right) & =2 \operatorname{Re}\left\{\boldsymbol{B}(\boldsymbol{\omega})\left\langle\frac{\partial \omega_{*}}{\partial x_{1}}\right\rangle \boldsymbol{f}(\boldsymbol{\omega})\right\},
\end{aligned}
$$

where 'Re' stands for 'the real part of', the superposed dot denotes differentiation with respect to time, and

$$
\begin{aligned}
\boldsymbol{t}_{2} & =\left(\sigma_{12}, \sigma_{22}, \sigma_{32}\right)^{\mathrm{T}}, \\
\boldsymbol{A}(\boldsymbol{\omega}) & =\left[\boldsymbol{a}_{1}\left(\omega_{1}\right), \boldsymbol{a}_{2}\left(\omega_{2}\right), \boldsymbol{a}_{3}\left(\omega_{3}\right)\right], \\
\boldsymbol{B}(\boldsymbol{\omega}) & =\left[\boldsymbol{b}_{1}\left(\omega_{1}\right), \boldsymbol{b}_{2}\left(\omega_{2}\right), \boldsymbol{b}_{3}\left(\omega_{3}\right)\right], \\
\boldsymbol{f}^{\prime}(\boldsymbol{\omega}) & =\left[f_{1}^{\prime}\left(\omega_{1}\right), f_{2}^{\prime}\left(\omega_{2}\right), f_{3}^{\prime}\left(\omega_{3}\right)\right]^{\mathrm{T}}
\end{aligned}
$$

and

$$
\begin{aligned}
\omega_{k} t & =x_{1}+p_{k}\left(\omega_{k}\right) x_{2}, \\
\frac{\partial \omega_{k}}{\partial x_{1}} & =\frac{1}{t-p_{k}^{\prime}\left(\omega_{k}\right) x_{2}},
\end{aligned}
$$


where the prime denotes differentiation with respect to the argument. In (2.1) and $(2.2),\left\langle h_{*}\right\rangle=\operatorname{diag}\left[h_{1}, h_{2}, h_{3}\right]$ represents a diagonal matrix. The scalar $p_{k}(\omega)$ and $\boldsymbol{a}_{k}(\omega)$ are, respectively, the eigenvalue and eigenvector of the following eigenvalue problem,

$$
\left[\boldsymbol{Q}+p\left(\boldsymbol{R}+\boldsymbol{R}^{\mathrm{T}}\right)+p^{2} \boldsymbol{T}-\rho \omega^{2} \boldsymbol{I}\right] \boldsymbol{a}(\omega)=\mathbf{0},
$$

where $\rho$ is the density and the matrices $\boldsymbol{Q}, \boldsymbol{R}$ and $\boldsymbol{T}$ are related to the elastic constants $C_{i j k s}$ by

$$
Q_{i k}=C_{i 1 k 1}, \quad R_{i k}=C_{i 1 k 2}, \quad T_{i k}=C_{i 2 k 2} .
$$

The vector $\boldsymbol{b}_{k}(\omega)$ is given by

$$
\boldsymbol{b}_{k}(\omega)=\left(\boldsymbol{R}^{\mathrm{T}}+p_{k}(\omega) \boldsymbol{T}\right) \boldsymbol{a}_{k}(\omega) .
$$

The eigenvalues $p_{k}(\omega), k=1,2,3$, are selected such that the imaginary parts are positive when they are complex. If $p_{k}(\omega)$ is real, $\omega=c, c$ being a constant, represents a line with slope $-1 / p_{k}(\omega)$ which intersects the $x_{1}$-axis at $x_{1}=c t$ for fixed $t$. The tangent point of the constant- $\omega$ line to the wavefront emanating from the origin after time $t$ is given by (Wu 2000)

$$
x_{1}=c t-p_{k}(c) x_{2}, \quad x_{2}=\frac{t}{p_{k}^{\prime}(c)} .
$$

Let the wavefront curve intersect the $x_{1}$-axis at $\pm c_{k} t$, determined by $\mathrm{d} \omega / \mathrm{d} p_{k}=0$. For $|c|>c_{k}$, there are two tangent lines passing through the point $x_{1}=c t$, one for which $x_{2}>0$ and the other $x_{2}<0$. Since each tangent line corresponds to a value of $p_{k}(\omega)$, the branch cuts from $c_{k}$ to $\infty$ and $-c_{k}$ to $-\infty$ in the complex $\omega$-plane should be introduced.

For isotropic media under plane-strain deformation, the eigenvalues $p_{1}(\omega)$ and $p_{2}(\omega)$ of $(2.5)$ can be easily obtained as

$$
p_{1}(\omega)=i \sqrt{1-\left(\frac{\omega}{c_{\mathrm{p}}}\right)^{2}}, \quad p_{2}(\omega)=\mathrm{i} \sqrt{1-\left(\frac{\omega}{c_{\mathrm{s}}}\right)^{2}},
$$

where $c_{\mathrm{p}}$ and $c_{\mathrm{s}}$ are, respectively, the dilatational wave and shear wave speeds. The corresponding eigenvectors $\boldsymbol{a}_{1}(\omega)$ and $\boldsymbol{a}_{2}(\omega)$ are

$$
\boldsymbol{a}_{1}(\omega)=\left(\begin{array}{c}
1 \\
p_{1}(\omega)
\end{array}\right), \quad \boldsymbol{a}_{2}(\omega)=\left(\begin{array}{c}
-p_{2}(\omega) \\
1
\end{array}\right) .
$$

The vectors $\boldsymbol{b}_{1}(\omega)$ and $\boldsymbol{b}_{2}(\omega)$ defined by $(2.6)$ are

$$
\boldsymbol{b}_{1}(\omega)=\rho c_{\mathrm{s}}^{2}\left(\begin{array}{c}
2 p_{1}(\omega) \\
p_{2}^{2}(\omega)-1
\end{array}\right), \quad \boldsymbol{b}_{2}(\omega)=\rho c_{\mathrm{s}}^{2}\left(\begin{array}{c}
1-p_{2}^{2}(\omega) \\
2 p_{2}(\omega)
\end{array}\right) .
$$

For a constant impulsive line force $\boldsymbol{F}_{0}^{*}$ applied at $x_{1}=\xi_{1}, x_{2}=\xi_{2}$ at $t=0$, the body force $\boldsymbol{F}^{*}$ may be expressed as $\boldsymbol{F}^{*}=\boldsymbol{F}_{0}^{*} \delta\left(x_{1}-\xi_{1}\right) \delta\left(x_{2}-\xi_{2}\right) \delta(t), \delta$ being the Dirac delta function. The corresponding expression for the displacement $\boldsymbol{u}^{*}$ may be expressed as (Wu 2000)

$$
\boldsymbol{u}^{*}\left(x_{1}, x_{2}, t\right)=\boldsymbol{G}^{*}\left(x_{1}-\xi_{1}, x_{2}-\xi_{2}, t\right) \boldsymbol{F}_{0}^{*},
$$


where

$$
\boldsymbol{G}^{*}\left(x_{1}-\xi_{1}, x_{2}-\xi_{2}, t\right)=-\frac{1}{\pi} \operatorname{Im}\left[\boldsymbol{A}(\boldsymbol{\omega})\left\langle\frac{1}{\gamma_{*}} \frac{\partial \omega_{*}}{\partial x_{1}}\right\rangle \boldsymbol{A}^{\mathrm{T}}(\boldsymbol{\omega})\right],
$$

where 'Im' stands for 'the imaginary part of', $\gamma_{k}=2 \boldsymbol{a}_{k}^{\mathrm{T}}\left(\omega_{k}\right) \boldsymbol{b}_{k}\left(\omega_{k}\right)$, and $\omega_{k}$ is determined by

$$
\omega_{k} t=x_{1}-\xi_{1}+p_{k}\left(\omega_{k}\right)\left(x_{2}-\xi_{2}\right) .
$$

\section{Non-uniform motion of a line force}

Consider a constant line force $\boldsymbol{F}_{0}$ which appears at $t=0$ suddenly at the origin and moves thereafter according to $x_{1}=\ell_{1}(t)$ and $x_{2}=\ell_{2}(t)$ with $\ell_{1}(0)=\ell_{2}(0)=0$. The corresponding body force may be described as $\boldsymbol{F}=\boldsymbol{F}_{0} \delta\left(x_{1}-\ell_{1}(t)\right) \delta\left(x_{2}-\ell_{2}(t)\right) H(t)$. The resulting displacement is denoted by $\boldsymbol{u}\left(x_{1}, x_{2}, t\right)$. Application of the reciprocal theorem to the present problem and that of an impulsive force discussed in the preceding section leads to

$$
\left(\boldsymbol{F}_{0}^{*}\right)^{\mathrm{T}} \boldsymbol{u}\left(\xi_{1}, \xi_{2}, t\right)=\int_{0}^{t} \boldsymbol{u}^{*}\left(\ell_{1}(\tau), \ell_{2}(\tau), t-\tau\right)^{\mathrm{T}} \mathrm{d} \tau \boldsymbol{F}_{0} .
$$

Substituting (2.11) into (3.1) and noting that $\boldsymbol{F}_{0}^{*}$ is arbitrary, we have

$$
\boldsymbol{u}\left(x_{1}, x_{2}, t\right)=G\left(x_{1}, x_{2}, t\right) \boldsymbol{F}_{0},
$$

where $\boldsymbol{G}\left(x_{1}, x_{2}, t\right)$ is the Green tensor given by

$$
\boldsymbol{G}\left(x_{1}, x_{2}, t\right)=\int_{0}^{t} \boldsymbol{G}^{*}\left(x_{1}-\ell_{1}(\tau), x_{2}-\ell_{2}(\tau), t-\tau\right) \mathrm{d} \tau .
$$

In (3.3), the variables $\xi_{i}$ have been changed to $x_{i}$ and the fact that

$$
\boldsymbol{G}^{*}\left(x_{1}-\ell_{1}(\tau), x_{2}-\ell_{2}(\tau), t-\tau\right)=\boldsymbol{G}^{*}\left(\ell_{1}(\tau)-x_{1}, \ell_{2}(\tau)-x_{2}, t-\tau\right)^{\mathrm{T}}
$$

has been used. The variable $\omega_{k}$ defined by (2.13) is now replaced by

$$
(t-\tau) \omega_{k}=x_{1}-\ell_{1}(\tau)+p_{k}\left(\omega_{k}\right)\left(x_{2}-\ell_{2}(\tau)\right)
$$

and $\partial \omega_{k} / \partial x_{1}$ by

$$
\frac{\partial \omega_{k}}{\partial x_{1}}=\frac{1}{t-\tau-p_{k}^{\prime}\left(\omega_{k}\right)\left(x_{2}-\ell_{2}(\tau)\right)} .
$$

The integral in (3.2) may be expressed in an alternative form as follows. From $(2.12), \boldsymbol{G}^{*}$ can be written as

$$
\boldsymbol{G}^{*}\left(x_{1}-\ell_{1}(\tau), x_{2}-\ell_{2}(\tau), t-\tau\right)=-\frac{1}{\pi} \operatorname{Im}\left[\sum_{k=1}^{3} \frac{\partial \omega_{k}}{\partial x_{1}} \frac{\boldsymbol{a}_{k}\left(\omega_{k}\right) \boldsymbol{a}_{k}^{\mathrm{T}}\left(\omega_{k}\right)}{\gamma_{k}\left(\omega_{k}\right)}\right] .
$$

From (3.4), for fixed $x_{1}, x_{2}$ and $t$ we may regard $\tau$ as a function of $\omega_{k}$ so that

$$
\frac{\partial \omega_{k}}{\partial x_{1}} \mathrm{~d} \tau=\frac{\mathrm{d} \omega_{k}}{\omega_{k}-\dot{\ell}_{1}-p_{k}\left(\omega_{k}\right) \dot{\ell}_{2}},
$$


where $\left(\dot{\ell}_{1}, \dot{\ell}_{2}\right)$ is the instantaneous force velocity. From (3.6) and (3.7), we have

$$
\boldsymbol{G}\left(x_{1}, x_{2}, t\right)=-\frac{1}{\pi} \operatorname{Im}\left[\sum_{k=1}^{3} \int_{\Gamma_{k}} \frac{1}{\omega_{k}-\dot{\ell}_{1}-p_{k}\left(\omega_{k}\right) \dot{\ell}_{2}} \frac{\boldsymbol{a}_{k}\left(\omega_{k}\right) \boldsymbol{a}_{k}^{\mathrm{T}}\left(\omega_{k}\right)}{\gamma_{k}\left(\omega_{k}\right)} \mathrm{d} \omega_{k}\right],
$$

where $\Gamma_{k}$ is the contour in the complex $\omega_{k}$-plane obtained from (3.4) as $\tau$ varies from 0 to $t$.

Although (3.2) is valid for arbitrary trajectories of force, only the case where the force starts from the origin and moves along the $x_{1}$-axis will receive further attention in the following sections. In this particular case, $\ell_{1}(t) \equiv \ell(t)$ and $\ell_{2}=0$.

\section{Wavefronts}

As the force moves along the $x_{1}$-axis, bulk wavelets are emitted from each point on the trajectory and propagate outward with the bulk wave speeds. The resulting wavefronts, where discontinuities or algebraic infinities in the solution occur, consist of the bulk wavefronts due to sudden application of the force at $(0,0)$ and possible Mach waves trailing the force (Freund 1972). The bulk wavefronts at a given time $t$ may be constructed using (2.7) with $c$ varying from $c_{k}$ to $\infty$. The Mach wavefronts are considered as follows.

In view of $(3.8)$, the condition for the existence of the Mach waves is $\omega_{k}=\dot{\ell}$ so that, from (3.4),

$$
(t-\tau) \dot{\ell}=x_{1}-\ell+p(\dot{\ell}) x_{2} .
$$

If $\dot{\ell}(\tau)>c_{k}$, or equivalently $p_{k}(\dot{\ell})$ is real, (4.1) represents a family of plane waves parametrized by $\tau$ for a given time $t$. The envelope of the above plane wavefronts may be expressed as

$$
\dot{x}_{1}(\tau)+p(\dot{\ell}) \dot{x}_{2}(\tau)=0 .
$$

By differentiating (4.1) with respect to $\tau$ and substituting (4.2), the Mach wavefront is obtained as

$$
x_{1}=\ell+(t-\tau) \dot{\ell}-p_{k}(\dot{\ell}) x_{2}, x_{2}=\frac{t-\tau}{p_{k}^{\prime}(\dot{\ell})},
$$

with $\tau$ ranging from 0 to $t$ subject to the condition that $p_{k}(\dot{\ell})$ is real. For isotropic materials (4.3) agrees with that derived by Freund (1972). It can be shown that the curvature $k_{m}$ of the Mach front is given by

$$
k_{m}=\left|\frac{p^{\prime} \ddot{\ell}}{\left(1+p^{2}\right)^{3 / 2}} \frac{1}{\dot{x}_{2}}\right|,
$$

where

$$
\dot{x}_{2}=-\frac{1+p^{\prime \prime} x_{2} \ddot{\ell}}{p^{\prime}} .
$$

The above equation indicates that the Mach front has cusps at the points where $\dot{x}_{1}=\dot{x}_{2}=0$.

If $\dot{\ell}(t)$ is constant, the wavefronts are obviously straight lines. The wavefronts are more complicated for non-uniform force motion. The wavefronts for two special cases of non-uniform motion of an in-plane force are shown in figures 1 and 2 for a cubic material. In figure $1, \ell(t)=a_{1} t^{2}$, and in figure $2, \ell(t)=a_{2} \sqrt{t}, a_{1}$ and $a_{2}$ being 


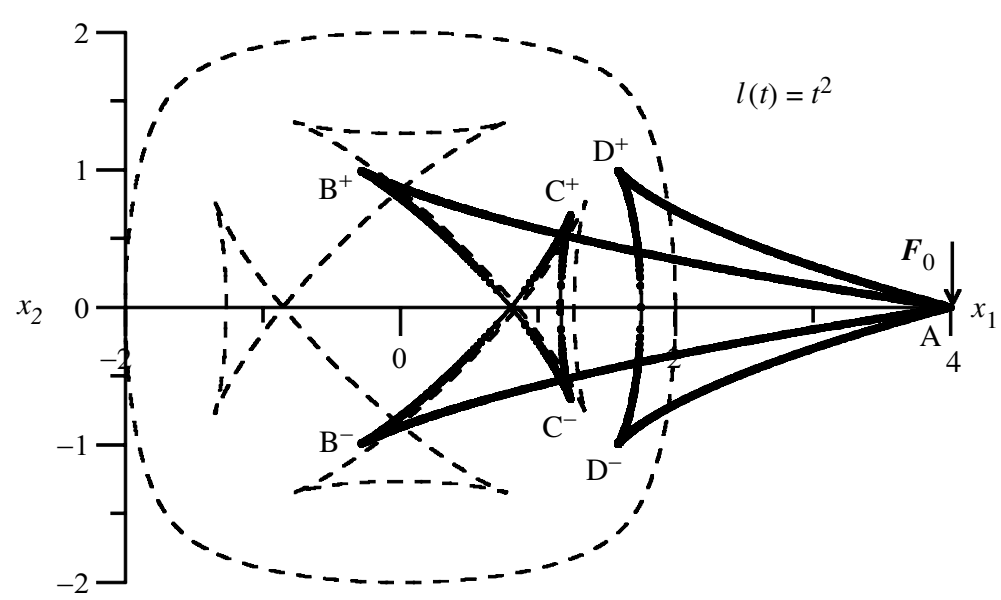

Figure 1. The wavefronts for an accelerating force in a cubic material.

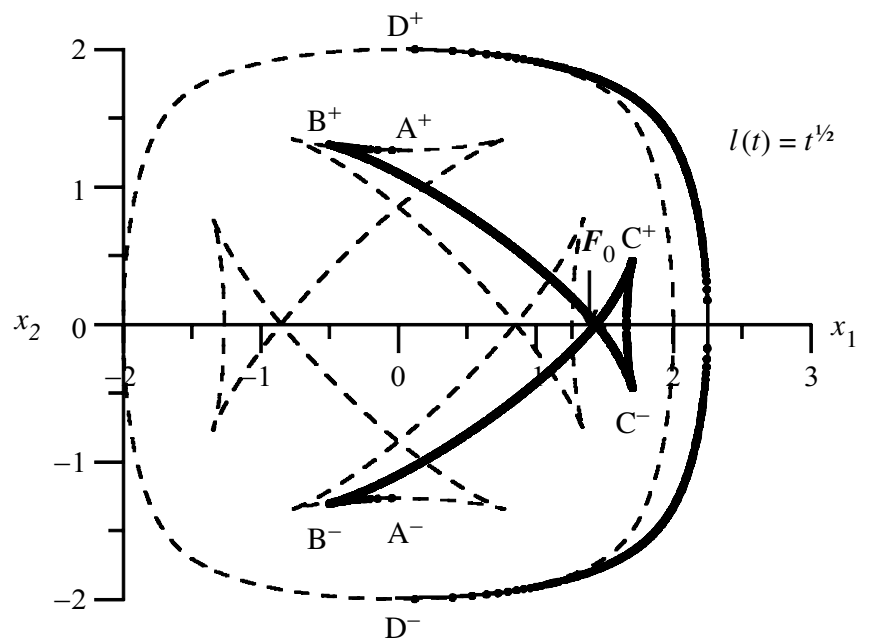

Figure 2. The wavefronts for a decelerating force in a cubic material.

constants. The same cases were considered for isotropic materials by Freund (1972). For convenience the units have been adjusted so that $a_{1}=a_{2}=\rho=1$ and the material constants are given by $C_{11}=C_{22}=1, C_{12}=0.8$ and $C_{66}=0.4$.

In the case of $\ell(t)=t^{2}$, the force accelerates from zero velocity to the sonic speeds of the material. The bulk wavefronts for $t=2$, shown as the dotted lines in figure 1 , consist of the $\mathrm{qL}$ (quasi-longitudinal) wavefront and the $\mathrm{qS}$ (quasi-shear) wavefront. The qS wavefront has four cuspidal triangles, two centred on the $x_{1}$-axis and two on the $x_{2}$-axis. The triangular regions are lacunas where there is no disturbance (Payton 1983). The qL wave speed $c_{1}$ in the $x_{1}$-direction is $c_{1}=1$, the fast qS wave speed $c_{2}=0.632$, and the slow qS wave speed $c_{3}=0.426$. The times at which the speed of the force are the bulk wave speed are $t=0.213$ for the slow $\mathrm{qS}$ wave and $t=0.25$ for the $\mathrm{qL}$ wave. The Mach wavefronts for $t=2$ were determined from (4.3) by varying $\tau$ from 0.213 to 2 for the qS Mach wavefront and from 0.25 to 2 for the $\mathrm{qL}$ Mach wavefront. The result is the curve $\mathrm{AB}^{-} \mathrm{C}^{+} \mathrm{C}^{-} \mathrm{B}^{+} \mathrm{A}$ for the $\mathrm{qS}$ Mach 
wave and $\mathrm{AD}^{-} \mathrm{D}^{+} \mathrm{A}$ for the $\mathrm{qL}$ Mach wave shown as the solid lines in figure 2. The segments $\mathrm{B}^{+} \mathrm{C}^{-}, \mathrm{B}^{-} \mathrm{C}^{+}$and $\mathrm{C}^{-} \mathrm{C}^{+}$lie closely behind the $\mathrm{qS}$ wavelet emanating from $x_{1}=0.0454$ corresponding to $\tau=0.213$. Similarly, the segment $\mathrm{D}^{-} \mathrm{D}^{+}$lies closely behind the $\mathrm{qL}$ wavelet emanating from $x_{1}=0.25$ corresponding to $\tau=0.5$. Similar features are also present for isotropic materials (Freund 1972).

For $\ell(t)=\sqrt{t}$, the wavefronts for $t=2$ are shown in figure 2 . The force decelerates continuously from a very large speed to 0.354 at $t=2$. The curve $\mathrm{D}^{-} \mathrm{D}^{+}$was determined from (4.3) by varying $\tau$ from 0 to 0.25 when the speed of the force is the $\mathrm{qL}$ wave speed and the curve $\mathrm{A}^{-} \mathrm{B}^{-} \mathrm{C}^{+} \mathrm{C}^{-} \mathrm{B}^{+} \mathrm{A}^{+}$from 0 to 1.38 when the speed is the slow qS wave speed. Either wavefront has separated from the moving force located at $x_{1}=\sqrt{2}$. Separation of the front from the force occurs when the force decelerates through a wave speed (Freund 1972).

\section{Asymptotic wavefront analysis}

In general, the displacement fields for non-uniform force motion can be obtained only by evaluating the integral of either (3.3) or (3.8) numerically. However, near the wavefronts analytic expressions can be derived by asymptotic analysis. Since the derivation is the same for each bulk wave, the subscripts used to distinguish different wave types are omitted for simplicity in the following discussions. Attention is restricted to a generic point in the first quadrant.

Consider the bulk wavefronts first. Let $\left(x_{1}, x_{2}\right)$ be a point behind the bulk wave excited by the force at $(0,0)$ at time $t$. From (3.6) the displacements due to the bulk wave may be expressed as

$$
\boldsymbol{u}=\operatorname{Im}\left[\int_{0}^{\tau_{0}} \frac{1}{g(\tau, \omega)} \frac{\boldsymbol{h}(\omega)}{\gamma(\omega)} \mathrm{d} \tau\right] H\left(\tau_{0}\right),
$$

where $H$ is the unit step function,

$$
\begin{aligned}
g(\tau, \omega) & =t-\tau-p^{\prime}(\omega) x_{2}, \\
\boldsymbol{h}(\omega) & =-\frac{1}{\pi}\left(\boldsymbol{a}^{\mathrm{T}}(\omega) \boldsymbol{F}_{0}\right) \boldsymbol{a}(\omega),
\end{aligned}
$$

$\omega$ is related to $\tau$ by $(3.4)$ and $g\left(\tau_{0}, \omega_{0}\right)=0$. The function $g$ can be approximated as

$$
g \simeq-\left(\tau-\tau_{0}\right)-p_{0}^{\prime \prime} x_{2}\left(\omega-\omega_{0}\right),
$$

where $p_{0}^{\prime \prime} \equiv p^{\prime \prime}\left(\omega_{0}\right)$. But by expanding (3.4) and using the fact that $g\left(\tau_{0}, \omega_{0}\right)=0$,

$$
\left(\dot{\ell}_{0}-\omega_{0}\right)\left(\tau-\tau_{0}\right)-\frac{1}{2} p_{0}^{\prime \prime} x_{2}\left(\omega-\omega_{0}\right)^{2} \simeq 0,
$$

where $\dot{\ell}_{0} \equiv \dot{\ell}\left(\tau_{0}\right)$, so that

$$
\omega-\omega_{0} \simeq\left(\frac{2\left(\dot{\ell}_{0}-\omega_{0}\right)\left(\tau-\tau_{0}\right)}{p_{0}^{\prime \prime} x_{2}}\right)^{1 / 2} .
$$

The square root should be taken such that the imaginary part of $\omega$ is positive. Hence

$$
g \simeq-p_{0}^{\prime \prime} x_{2}\left(\frac{2\left(\dot{\ell}_{0}-\omega_{0}\right)\left(\tau-\tau_{0}\right)}{p_{0}^{\prime \prime} x_{2}}\right)^{1 / 2} .
$$


Substitution of (5.6) into (5.1) then yields

$$
\boldsymbol{u} \simeq \operatorname{Re}\left[\frac{1}{p_{0}^{\prime \prime} x_{2}}\left(\frac{2 p_{0}^{\prime \prime} x_{2}\left(t-t_{0}\right)}{\left(\dot{\ell}_{0}-\omega_{0}\right)}\right)^{1 / 2} \frac{\boldsymbol{h}\left(\omega_{0}\right)}{\gamma\left(\omega_{0}\right)}\right] H\left(t-t_{0}\right),
$$

where $t_{0}=t-\tau_{0}$ is the time for the bulk wavelet to propagate from $\left(\ell\left(\tau_{0}\right), 0\right)$ to the point of interest.

Equation (5.7) is also not valid if $p_{0}^{\prime \prime}=0$. It can be shown that the curvature of the bulk wavefront $k_{\mathrm{b}}$ is given by

$$
k_{\mathrm{b}}=\left|\frac{p^{\prime 3}}{\left(1+p^{2}\right)^{3 / 2}} \frac{1}{p^{\prime \prime}}\right|
$$

Thus the points where $p_{0}^{\prime \prime}=0$ appear as cusps on the wavefront. At these cuspidal points $g$ may be approximated as

$$
g \simeq-\left(\tau-\tau_{0}\right)-\frac{1}{2} p_{0}^{\prime \prime \prime} x_{2}\left(\omega-\omega_{0}\right)^{2},
$$

where $p_{0}^{\prime \prime \prime}=p^{\prime \prime \prime}\left(\omega_{0}\right)$ and (3.4) may be expanded as

$$
\left(\dot{\ell}_{0}-\omega_{0}\right)\left(\tau-\tau_{0}\right)-\frac{1}{6} p_{0}^{\prime \prime \prime} x_{2}\left(\omega-\omega_{0}\right)^{3} \simeq 0 .
$$

The corresponding displacements become

$$
\boldsymbol{u} \simeq \operatorname{Im}\left[\frac{6}{p_{0}^{\prime \prime \prime} x_{2}}\left(\frac{p_{0}^{\prime \prime \prime} x_{2}}{6\left(\dot{\ell}_{0}-\omega_{0}\right)}\right)^{2 / 3} \frac{\left(t_{0}-t\right)^{1 / 3}}{\gamma\left(\omega_{0}\right)} \boldsymbol{h}\left(\omega_{0}\right)\right] H\left(t-t_{0}\right)
$$

The Mach wavefront is discussed next. Let $t_{*}$ be the time for the Mach wave to arrive at a generic point $\left(x_{1}, x_{2}\right)$. From (4.3) the arrival time $t_{*}$ is determined by

$$
t_{*}=\tau_{*}+p^{\prime}\left(\dot{\ell}_{*}\right) x_{2},
$$

where $\dot{\ell}_{*} \equiv \dot{\ell}\left(\tau_{*}\right)$. From (3.6) the displacements due to the Mach wave at $t \rightarrow t_{*}$ may be expressed as

$$
\boldsymbol{u}=\operatorname{Im}\left[\int_{\tau_{*}-\varepsilon}^{\tau_{*}+\varepsilon} \frac{1}{g(\tau, \omega)} \frac{\boldsymbol{h}(\omega)}{\gamma(\omega)} \mathrm{d} \tau\right] H\left(t-t_{*}\right)
$$

where $\varepsilon$ is a small positive number and $g\left(\tau_{*} \pm \varepsilon, \omega\right)=0$. For $\left|\tau-\tau_{*}\right|<\varepsilon$ the function $g(\tau, \omega)$ can be approximated as

$$
g(\tau, \omega) \simeq t-t_{*}-\left(\tau-\tau_{*}\right)-p_{*}^{\prime \prime} x_{2}\left(\omega-\dot{\ell}_{*}\right)
$$

However, by expanding (3.4) and using (5.11)

$$
\left(t-t_{*}\right) \dot{\ell}_{*}+\ddot{\ell}_{*}\left(\tau-\tau_{*}\right)^{2}-2\left(\tau-\tau_{*}\right)\left(\omega-\dot{\ell}_{*}\right)-p_{*}^{\prime \prime} x_{2}\left(\omega-\dot{\ell}_{*}\right)^{2} \simeq 0,
$$

where $\ddot{\ell}_{*} \equiv \ddot{\ell}\left(\tau_{*}\right)$, or

$$
\omega-\dot{\ell}_{*} \simeq-\frac{1}{p_{*}^{\prime \prime} x_{2}}\left(\tau-\tau_{*}+\sqrt{\left(1+\ddot{\ell}_{*} p_{*}^{\prime \prime} x_{2}\right)\left(\tau-\tau_{*}\right)^{2}+p_{*}^{\prime \prime} \dot{\ell}_{*} x_{2}\left(t-t_{*}\right)}\right) .
$$

Again the square root should be taken such that the imaginary part of $\omega$ is positive. By substituting (5.14) into (5.13),

$$
g \simeq \sqrt{1+\ddot{\ell}_{*} p_{*}^{\prime \prime} x_{2}} \sqrt{\left(\tau-\tau_{*}\right)^{2}-\varepsilon^{2}},
$$


where

$$
\varepsilon=\sqrt{p_{*}^{\prime \prime} \dot{\ell}_{*} x_{2}\left(t_{*}-t\right) /\left(1+\ddot{\ell}_{*} p_{*}^{\prime \prime} x_{2}\right)}
$$

and $1+\ddot{\ell}_{*} p_{*}^{\prime \prime} x_{2}>0$ is assumed. From (4.5) the assumption holds if $\dot{x}_{2}<0$. With (5.15), (5.12) yields

$$
\boldsymbol{u} \simeq-\pi \operatorname{Re}\left[\frac{1}{\sqrt{1+\ddot{\ell}_{*} p_{*}^{\prime \prime} x_{2}}} \frac{\boldsymbol{h}\left(\dot{\ell}_{*}\right)}{\gamma\left(\dot{\ell}_{*}\right)}\right] H\left(t-t_{*}\right) .
$$

Note that the displacement contains a factor which is proportional to the square root of the curvature. The factor is unity for the case of a constant velocity force.

\section{Line force moving with constant velocity}

Consider a line force $\boldsymbol{F}_{0}$ applied suddenly at the origin and moving thereafter with constant velocity $v$. The corresponding Green tensor is given by setting $\dot{\ell}=v$ in (3.8) as

$$
\boldsymbol{G}\left(x_{1}, x_{2}, t\right)=-\frac{1}{\pi} \operatorname{Im}\left[\sum_{k=1}^{3} \int_{\omega_{k}^{(0)}}^{\infty} \frac{1}{\omega_{k}-v} \frac{\boldsymbol{a}_{k}\left(\omega_{k}\right) \boldsymbol{a}_{k}^{\mathrm{T}}\left(\omega_{k}\right)}{\gamma_{k}\left(\omega_{k}\right)} \mathrm{d} \omega_{k}\right],
$$

where $\omega_{k}^{(0)}=x_{1} / t+p_{k}\left(\omega_{k}^{(0)}\right) x_{2} / t$. Note that the integrand in the preceding equation is a function of $\omega_{k}$ only so that the time derivative of $\boldsymbol{G}$ can be obtained explicitly as

$$
\begin{aligned}
\dot{\boldsymbol{G}}\left(x_{1}, x_{2}, t\right)=-\frac{1}{\pi} \operatorname{Im}\left[\boldsymbol{A}\left(\boldsymbol{\omega}^{(0)}\right)\right. & \left.\left\langle\frac{\omega_{*}^{(0)}}{\omega_{*}^{(0)}-v} \frac{1}{\gamma_{*}\left(\omega_{*}^{(0)}\right)} \frac{1}{t-p_{*}^{\prime}\left(\omega_{*}^{(0)}\right) x_{2}}\right\rangle \boldsymbol{A}^{\mathrm{T}}\left(\boldsymbol{\omega}^{(0)}\right)\right] \\
& \pm v \operatorname{Re}\left[\boldsymbol{A}^{ \pm}(v)\left\langle\frac{\delta\left(\omega_{*}^{(0)}-v\right)}{\gamma_{*}^{ \pm}(v)} \frac{1}{t-p_{*}^{ \pm \prime}(v) x_{2}}\right\rangle \boldsymbol{A}^{ \pm \mathrm{T}}(v)\right]
\end{aligned}
$$

where the plus sign before the second term is for $x_{2}>0$, minus for $x_{2}<0$ and the superscripts + and - denote the limits as $x_{2} \rightarrow 0^{+}$and $x_{2} \rightarrow 0^{-}$, respectively. In deriving (6.2), the following relation has been used:

$$
\frac{\partial \omega_{k}^{(0)}}{\partial t}=-\frac{\omega_{k}^{(0)}}{t-p_{k}^{\prime}\left(\omega_{k}^{(0)}\right) x_{2}}
$$

The first term on the right-hand side of (6.2) is due to the bulk waves, while the second term is associated with the Mach waves. For a stationary force, $v=0$ and (6.2) reduces to $(2.12)$ as it should. In addition, as the moving force is approached, i.e. $x_{1} \rightarrow v t$ and $x_{2} \rightarrow 0$, the steady-state solution (Stroh 1962) is recovered.

For $x_{2}=0, \omega_{k}^{(0)}=x_{1} / t$ and (6.2) may be simplified as

$$
\dot{\boldsymbol{G}}\left(x_{1}, t\right)=\frac{1}{1-v t / x_{1}} \frac{1}{2 \pi t} \operatorname{Re}\left[\boldsymbol{H}\left(\frac{x_{1}}{t}\right)\right]+\frac{1}{2} \delta\left(t-\frac{x_{1}}{v}\right) \operatorname{Im}\left[\boldsymbol{H}^{+}(v)\right],
$$

where $\boldsymbol{H}(v)$ is defined as (Ting 1996, p. 446)

$$
\boldsymbol{H}(v)=2 \mathrm{i} \boldsymbol{A}(v)\left\langle\frac{1}{\gamma_{*}(v)}\right\rangle \boldsymbol{A}^{\mathrm{T}}(v) .
$$


It can be shown that the symmetric matrix $\boldsymbol{H}(v)$ is real, complex or imaginary if $v$ is subsonic, transonic or supersonic, respectively. Moreover,

$$
\boldsymbol{H}^{-}(v)=\overline{\boldsymbol{H}}^{+}(v) .
$$

For orthotropic materials $\boldsymbol{H}(v)$ is a diagonal matrix with the diagonal elements given explicitly as (Ting 1996, p. 480)

$$
\begin{gathered}
\sqrt{C_{11}^{*} C_{66}} H_{11}=\sqrt{C_{66}^{*} C_{22}} H_{22}=\left(\sqrt{C_{11}^{*} C_{22}}+\sqrt{C_{66}^{*} C_{66}}\right) \frac{1}{\sqrt{Y}}, \\
H_{33}=\frac{1}{\sqrt{C_{55}^{*} C_{44}}},
\end{gathered}
$$

where

$$
Y(v)=\left(\sqrt{C_{11}^{*} C_{22}}+\sqrt{C_{66}^{*} C_{66}}\right)^{2}-\left(C_{12}+C_{66}\right)^{2},
$$

$C_{i j}^{*}=C_{i j}-\rho v^{2} \delta_{i j}$ and $C_{i j}$ is the contracted notation for the elastic constants. For isotropic materials (6.4) and (6.5) become

$$
q_{1} H_{11}=q_{2} H_{22}=\frac{\left(1-q_{2} q_{1}\right)}{\rho c_{\mathrm{s}}^{2}\left(1-q_{2}^{2}\right)}, \quad H_{33}=\frac{1}{\rho c_{\mathrm{s}}^{2} q_{2}},
$$

where $q_{1}(v)=\sqrt{1-\left(v / c_{\mathrm{p}}\right)^{2}}, q_{2}(v)=\sqrt{1-\left(v / c_{\mathrm{s}}\right)^{2}}$.

Figure 3 shows plots of $\dot{G}_{22}$ for some $x_{1}>0$ and $x_{2}=0$ and for various values of $v$. The result is expressed in terms of the following dimensionless quantities:

$$
g^{*}=\frac{2 \pi C_{11} x_{1}}{c_{1}} \dot{G}_{22}, \quad t^{*}=\frac{c_{1} t}{x_{1}},
$$

where $c_{1}=\sqrt{C_{11} / \rho}$. The calculations were carried out for the cubic material considered in the preceding section. The values of $v$ selected were $(a) v=0$ (stationary), (b) $v=0.3 c_{1}$ (subsonic), (c) $v=0.8 c_{1}$ (transonic) and $(d) v=1.5 c_{1}$ (supersonic). For all cases $\dot{G}_{22}$ is singular upon the arrival of the bulk waves. In addition, as the force arrives $\dot{G}_{22}$ exhibits a simple pole singularity for case $(b)$, whereas a $\delta$-singularity is generated for case $(d)$. For case $(c)$ a pole as well as a $\delta$-singularity is produced.

\section{Concluding remarks}

An exact integral solution of the displacements has been derived for a line force moving non-uniformly in an unbounded elastic body of general anisotropy. The wavefronts, especially the Mach waves, have been discussed, and first motion responses have been determined immediately behind the wavefronts. For the special case of a constant force speed, a closed-form solution of the particle velocities has been obtained. The method of solution presented here may also be used to study the problem of a non-uniformly moving force on an elastic half-space. The work is in progress and will be reported in a separate communication.

The research was supported by the National Science Council of Taiwan under grant NSC 892212-E-002-007. 

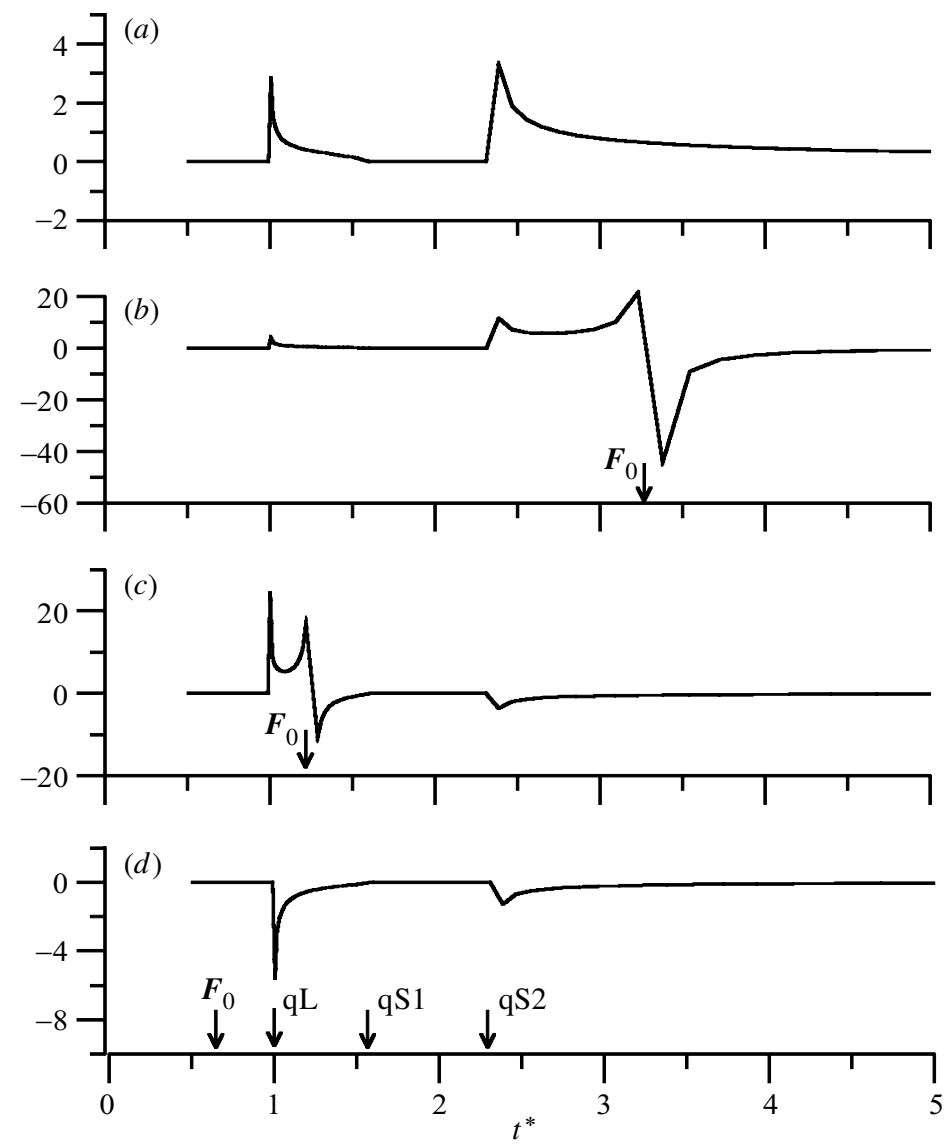

Figure 3. $\dot{G}_{22}$ as a function of $t$ at a point on the $x_{1}$-axis in a cubic material due to a force moving with speed $v=0(a), 0.3 c_{1}(b), 0.8 c_{1}(c), 1.5 c_{1}(d)$.

\section{References}

Ang, D. D. 1960 Transient motion of a line load on the surface on an elastic half space. Q. Appl. Math. 18, 251-256.

Cole, J. D. \& Huth, J. 1958 Stresses produced in a half plane by moving loads. ASME J. Appl. Mech. 28, 433-436.

Eason, G., Fulton, J. \& Sneddon, I. N. 1956 The generation of waves in an elastic solid by variable body forces. Phil. Trans. R. Soc. Lond. A 248, 575.

Freund, L. B. 1972 Wave motion in an elastic solid due to a non-uniformly moving line load. $Q$. Appl. Math. 30, 271-281.

Freund, L. B. 1973 The response of an elastic solid to non-uniformly moving surface loads. ASME J. Appl. Mech. 40, 699-704.

Gakenheimer, D. C. \& Miklowitz, J. 1969 Transient excitation of an elastic half space by a point load travelling on the surface. ASME J. Appl. Mech. 36, 505-515.

Payton, R. G. 1967 Transient motion of an elastic half space due to a moving surface line load. Int. J. Engng Sci. 5, 49-79.

Payton, R. G. 1968 Epicenter motion of an elastic half-space due to buried stationary and moving line sources. Int. J. Solids Struct. 4, 287-300. 
Payton, R. G. 1983 Elastic wave propagation in transversely isotropic media. The Hague: Martinus Nijhoff.

Sneddon, I. N. 1951 Fourier transforms, pp. 445-449. McGraw-Hill.

Stroh, A. N. 1962 Steady state problems in anisotropic elasticity. J. Math. Phys. 41, 77-103.

Ting, T. C. T. 1996 Anisotropic elasticity - theory and application. Oxford University Press.

Wu, K.-C. 2000 Extension of Stroh's formalism to self-similar problems in two-dimensional elastodynamics. Proc. R. Soc. Lond. A 456, 869-890. 